\title{
Regularities in discrete hierarchy seismo-acoustic mode in a geophysical field
}

\author{
Kamel Baddari ${ }^{1, \star}$ and Anatoly D. Frolov ${ }^{2}$ \\ ${ }^{1}$ Laboratory of Physics of the Earth, University of Boumerdes, Algeria \\ ${ }^{2}$ Russian Academy of Sciences, Geophysical Division NCG, Moscow, Russia
}

\author{
Article history \\ Received May 13, 2010; accepted October 9, 2010. \\ Subject classification: \\ Geophysical field, Seismo-acoustic regime, Auto-similarity, Fractal structure.
}

\section{ABSTRACT}

Some regularities in seismo acoustic mode have been studied during the preparation and development of dynamic events generated during the deformation at different scales in a geophysical field. The time-space behavior of certain auto similarity parameters: the slope of the recurrence plot $\gamma$, the fractal dimension of the hypocenters set $\mathrm{D}$, the relationship D-3 $\gamma$ and the crack concentration parameter $\mathrm{K}_{\mathrm{sr}}$ in laboratory and field experiments in Algerian seismoactive zone have been analyzed as well. Precursory stage and local failure evolution in rock samples and in natural conditions were studied. It is shown that the regularities in the behavior of the parameters under study do not qualitatively depend on the dimension of the object being loaded. The quoted examples above speak of inhomogeneity and self-similarity of seismicity distribution in space-time. This suggests that the evolution of cracking process at different scales, from rock samples to Earth crust, is controlled by the same physical mechanism. The further development of these studies consolidates the physical basis of the prediction of the dynamic events.

\section{Introduction}

A geophysical field is a set of geophysical properties of rock at a level ranging from a rock sample scale to a planet scale [Sadovsky and Pisarenko 1991, Lockner et al. 1992, Baddari and Frolov 1997, Sobolev and Ponomarev 2003, Vettegren et al. 2004, Thompson et al. 2006, Eftaxias et al. 2007, LiakopoulouMorris et al. 2007, Smirnov et al. 2010]. The seismic structure is distinguished by its auto-similarity property in the spatial, temporal and energetic domains [Gutenberg 1956, Smirnov et al. 1995, Baddari et al 1996, Baddari et al. 1999, Sobolev and Ponomarev 1999, Zang et al. 2000, Kanamori 2004, Lei and Satoh 2007, Kuksenko et al. 2009]. The nature of this structure is not defined clearly in situ because of a lack of reliable experimental data. Modeling in a laboratory using the inverseloading technique by acoustic emission has allowed the final phase of source development to be extended and the acoustic regime to be analyzed in detail [Lockner 1993, Sobolev et al.
1996, Baddari 1997, Smirnov 1997, Lei et al. 2004, Smirnov and Ponomarev 2004, Amitrano 2006, Xu et al. 2009, Yuan and Li 2009, Dresen et al. 2010, Youn and Tonon 2010]. This study analyzes the similarity of the dynamic seismo-acoustic regime at different scales of a geophysical field.

\section{Parameters of the seismo-acoustic regime}

\subsection{Relationship between}

the recurrence plot slope $\gamma$ and the fractal dimension $\mathrm{D}$

Independent of its scale, rock failure is a multi-stage process, and the final stages can take different forms [Sadovsky and Pisarenko 1991]. The fracture results from shear stress, which accumulates during tectonic deformation. A tectonic earthquake can be considered as a final step in a determined phase of a discrete failure stochastic process in the Earth crust. This process leads to total or partial stress release over the fracture area, and continues to be developed in different stress fields. Pore pressure and effective normal stress evolution affect the dynamic propagation of the earthquake rupture [Bizzarri and Cocco 2006].

We consider a seismic source of a size $L_{i}$ that gives rise to a stress drop in its neighboring space of linear dimension $X$ [Smirnov et al. 1995, Baddari and Djeddi 2009]:

$$
X=\rho L_{i}^{c}
$$

After the earthquake, this creates a seismic quiescence, i.e. no earthquake with the same source characteristics can occur in the same domain, during the period $\Delta \mathrm{t}$ :

$$
\Delta t=\psi L_{i}^{\zeta}
$$

where $\rho, c, \psi$, and $\zeta$ are specific constants [Smirnov 1995].

Taking into account the fractal property of a geophysical medium, we consider that the space of a seismogenic zone 
of size $Y$ can be composed of a subspace set of size $R_{i}$. The number $j$ of the $R_{i}$-sized subspaces, which contain earthquakes, will be equal to:

$$
j=\left(Y / R_{i}\right)^{D}
$$

where $D$ is the fractal dimension of the seismogenic zone.

At the same time, the seismic cycle $t$ is also characterized by a fractal structure, which can be divided, and will contain $p$ nonempty subintervals:

$$
p=(t / \Delta t)^{D_{t}}
$$

where the parameter $D_{t}$ has the meaning of a temporal fractal dimension.

The seismic energy, as a function of its source size [Sadovsky et al. 1991], is given by:

$$
E_{s}=a L_{i}^{\alpha}
$$

with $\alpha=3$ on average [Kasahara 1985].

Therefore, if $R_{i}=X$, we will have the number of events with source size $L_{i}$ that occur in a space of size $Y$ during time $t$ equal to:

$$
N=\left(Y / R_{i}\right)^{D}(t / \Delta t)^{D_{t}}
$$

If we substitute Equations (1), (2) and (5) into Equation (6), we arrive at the seismic recursive general law of the fractal property of seismicity:

$$
\log N=-\gamma \log E_{s}+D \log Y+D_{t} \log t+\tau,
$$

where:

$$
\begin{aligned}
\tau & =\gamma \log a-D \log \rho-D_{t} \log \psi, \\
\gamma & =\frac{c D+\zeta D_{t}}{\alpha} .
\end{aligned}
$$

Thus, we can deduce the following relationship between the recurrence plot slope $\gamma$, the fractal dimension $D$, and the temporal fractal dimension $D_{t}$ :

$$
\alpha \gamma-c D-\zeta D_{t}=0
$$

Taking $\alpha=3$ in Equation (8) and assuming $c=1$ [Smirnov et al. 1995], we get:

$$
D=3 \gamma-\zeta D_{t}
$$

Experimentally, $\gamma \approx 0.5$ and $D \approx 1.5$ on average [Smirnov 1993, Baddari and Frolov 1997, Smirnov and Ponomarev 2004, Liu et al. 2009], and therefore we can deduce that the constant $\zeta \approx 0$ in Equation (9), since $D_{t}>0\left(D_{t}=0\right.$ means that all earthquakes will occur simultaneously). The equality of Equation (9) is equivalent to $D_{t}=2 \gamma_{M}$, where $\gamma_{M}$ is the slope of the recurrence curve for magnitudes $\left(\gamma_{M}=1.5 \gamma\right)$ [Main et al. 1990].

\subsection{Crack concentration parameter $\mathrm{K}_{\mathrm{sr}}$}

The transition from microcracks to macrocracks in a loaded object of volume $v_{0}$ takes place upon reaching a certain critical concentration of cracks in the failure source of the appropriate dimensions. The crucial factor here is the crack concentration parameter, which is calculated using the formula [Baddari et al. 1996, Zavyalov 2006]:

$$
K_{s r}=q^{-1 / 3} / \bar{L}
$$

where $q^{-1 / 3}=N / v_{0}$ is the volume density of the acoustic (or seismic) events that occur in the object, and $\bar{L}$ is the average rupture length. The dimensionless parameter $K_{s r}$ reflects the ratio of the mean distance between ruptures and the average rupture length.

The average rupture length in the crack set is:

$$
\bar{L}=1 / N \sum_{i=1}^{N} L_{i}
$$

where $N$ is the number of acoustic (or seismic) events of energy class $k_{i} \in\left[k_{\min }-k_{\max }\right]$ appearing in volume $v_{0}$ during the time $\Delta t, L_{i}$ is the size of the opening of microcracks or the unity rupture length:

$$
\log L_{i}=\varepsilon k_{i}+\delta
$$

here, $\varepsilon=0.244, \delta=-2.266$ for the energy classes $k_{i}$, and $\varepsilon=$ $0.440, \delta=-1.289$ for the magnitudes $M\left(k_{i}=0.244+1.8 \mathrm{M}\right)$ [Sobolev and Ponomarev 2003].

The parameter $K_{s r}$ represents the ability of cracks to interact and develop into larger defects, i.e. when the mean distance between cracks becomes equal to a critical value, a macrocrack is formed that causes the development of damage in the rock.

\section{Experimental technique and methods of original data analysis}

Acoustic emissions by rock samples under failure reflect the process of crack formation and can be considered as a model for the seismicity in the Earth crust [Han and Yang 2009, Senfaute et al. 2009]. To determine the auto-similarity of the seismo-acoustic regime at different scales, an unparalleled series of six rock experiments (BF1-6) were carried out under bi-axial compression conditions on models with two layers, using surfaces of $500 \times 260 \mathrm{~mm}$, with the lower layer of dry granite of $420 \mathrm{~mm}$ thickness, surmounted by a layer of dry sandstone (or concrete) of $80 \mathrm{~mm}$ (Figure 1). 
A petrographic analysis gave the granite composition as follows: $42 \%$ albite, $42 \%$ quartz, $39 \%$ biotite, and an admixture of muscovite, pyrite and other components. The grain size was 1-2 $\mathrm{mm}$. The lower layer was heated up to $400^{\circ} \mathrm{C}$. The objective was to reconcile this with the natural conditions of the superficial Algerian seismic events. The formation of the main rupture during the last stage of the process was extended by the addition of a maximum number of microcracks under quasi-static conditions for a few hours, by decreasing the rate of axial deformation once the crack acceleration started. This allows the rheologic state to be reached during the long period of a deformation process. Two stress concentrators introduced in the model at a $35^{\circ}$ angle, with respect to the direction of intermediate vertical charge $H$, encouraged the cutting (shear fracture) of the lower layer, which then propagated to the upper layer block at an angle of $30^{\circ}$ to $45^{\circ}$. An intense evolution of deformations starting from concentrator extremities was noted, which was due to the interactions of their stress fields. The intermediate load $H$ was maintained practically constant at $H=50 \mathrm{MPa}$. The mean load $F$ applied to the granite changed over three cycles: (i) $F$ gradually increased up to a maximum $\left(F_{\max }\right)$ of typically (0.4-0.5) $t / t_{f}$, where $t$ is the experimental time, given with respect to the total life-time $t_{f}$ of the experiment; (ii) $F$ was maintained approximately constant up to $(0.75-0.8) t / t_{f}$; and (iii) $F$ decreased in the interval $[(0.75-0.8)-1] t / t_{f}$ and then the main rupture of the block began to appear.

Acoustic signals were detected by resonance piezoelectric transducers of eigen frequency of $0.2 \mathrm{MHz}$. A special multichannel system was used to record the wave forms. The block surfaces were covered with eight sensors linked to input amplifiers, GRS-6052 oscillographs and a computer. A transducer was fixed in the middle of the sample to serve as a controller of the organization of the inverse commands of the piston pressure, and as a means to measure the loading as a function of the acoustic activity. The loading was realized under the condition of invariability of the acoustic event intensity. The acoustic event activity used in the chain of the inverse command was of the first dozens of events per second. Exceeding the acoustic event rate activity immediately led to a decrease in the axial load through a reduction in the motion speed of the piston pressure. The strain-control regime was selected in agreement with the concept relative to the process that occurs in the earthquake focal zones. The constant rate of strain, $5 \mathrm{MPa} / \mathrm{s}$, and the ability to vary this during the experiment, allowed control of the stability of the development of the macrofailure in the sample. The load $F$ was measured at a rate of 1-s sampling on a personal computer.

The recording system of the acoustic events was realized by recording the times of the first arrivals and the first maximum amplitudes of the signals of each transducer, under a numerical format. The resolution of the numerical

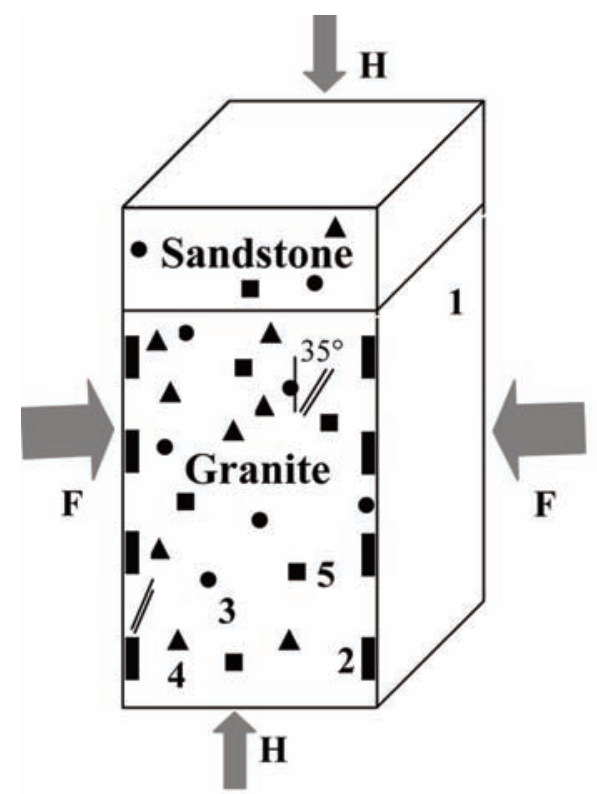

Figure 1. Schematic diagram of the experiment arrangement. (1) Pattern with artificial stress concentrators; (2) acoustic emission sensors (rectangles); (3) apparent resistivity electrodes (circles); (4) self-potential electrodes (triangles); (5) ultrasonic transducers (squares); F, main load; H, intermediate load.

channel for the determination of the arrival time was of the order of $0.04 \mu$ s to $0.05 \mu$ s.

To pinpoint acoustic event sources, a seismological technique was used to find the hypocenter coordinates, through the differences in the acoustic event signals registered over time. We applied an algorithm using the time of the first arrivals of longitudinal waves to the transducer and the minimum propagation time. Acoustic events with propagation times greater than $5 \mu$ s were eliminated from the acoustic event catalog. Nevertheless, the coordinates of the sources of an acoustic event were determined with an error of $1.0 \mathrm{~mm}$ to $1.5 \mathrm{~mm}$, according to the acoustic event value.

The calculations were realized in windows of 1,000 events, which were varied by steps of 500 . The number of acoustic event coordinates determined for the experiments BF1-BF6 were, respectively, 37396, 48332, 42785, 36120, 44280 and 36648 , which represents between $48.4 \%$ and $38.4 \%$ of the total of the recorded signals.

The catalog of the acoustic events comprises time, the Cartesian coordinates, and the energy class $k_{i}$ of the event $\left(k_{i}=2 \lg A\right.$, where $A$ is the amplitude of the impulse in $\mathrm{mV}$ brought to the $10 \mathrm{~mm}$ distance from the hypocenter). In this case, $k_{i}$ is similar to the energy class used in seismology.

We selected the acoustic events with regard to their representative energy class $k_{i}$, which was estimated from the analysis of the recurrence plots $N\left(k_{i}\right)=A 10^{-\gamma\left(k_{i}-2\right)}$ (Figure 2). The values of the minimal energy class, $k_{\text {min }}$, were $1.7,1.8,1.9$ and 1.5, for the experiments BF1, BF2, BF3 and BF4, respectively. The square of the amplitude of the acoustic pulses $\left(A^{2}\right)$ is proportional to the energy of an event, within a certain allowance.

The control of the anisotropy failure evolution structure 


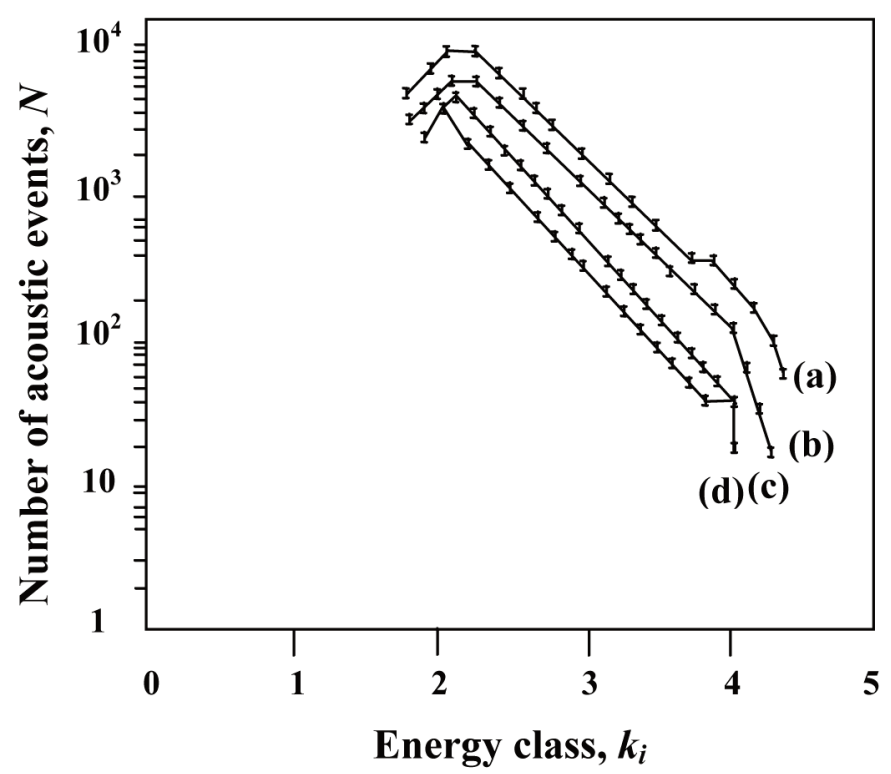

Figure 2. Recurrence curves of the acoustic events in the experiments. (a) $\mathrm{BF} 1$; (b) BF2; (c) BF3; (d) BF4.

elements was realized by studying the elastic longitudinal and transversal wave velocities $\left(V_{P}\right.$ and $\left.V_{S}\right)$, the electric apparent resistivity $\Delta \rho_{a}$, and the spontaneous electric polarization $\Delta U$.

To study the variations in the elastic-wave velocities, each free face of a block had eight piezo-electric compressional transducers with a free resonance frequency from $80 \mathrm{kHz}$ to $100 \mathrm{kHz}$. These were used as focuses and receivers of ultrasonic signals. In the experiments, mainly longitudinal wave transducers were used, but these can easily be replaced by $S$-wave transducers. Ultrasonic sounding started under noload conditions, but as the cycled loading progressed, a series of measurements were taken along the routes. The number of monitored paths was 27 ; this enables the study of velocity variations in measuring the $P$-wave and $S$-wave arrival times of about $0.7 \%$, and for an amplitude of $10 \%$. The arrival time of the first break was used to calculate the ultrasonic velocities. The elastic-wave velocities were measured following the ultrasound traces at the angles of $25^{\circ}, 45^{\circ}$ and $75^{\circ}$, with respect to the vertical axis of the sample.

The sensors of electric potential were nonpolarizable chlorine-silver electrodes. They were placed on the free faces of the sample. Various combinations of pick-offs served as receiving pairs of electrodes $\mathrm{MN}$, which allowed the study of the structure of the electric field in detail. The intrinsic drift of the electrode potentials did not exceed $0.6 \mathrm{mV}$ for a complete loading cycle. The background values had been estimated prior to the experiment, by carrying out an areal survey of the surface potentials on a $2 \times 2 \mathrm{~cm}$ grid on all of the faces of the sample.

The variations in apparent electric resistivity were studied using direct current and four-electrode devices under different orientations. The receiving dipoles were various combinations of the nonpolarizable sensors mentioned above. This strength allowed us to evaluate relative changes of resistivity both in the entire block and in separate parts of it. The uncertainty of measuring the variations of apparent resistivity was below $2 \%$.

For the seismic events, we considered the catalogs of seismicity of north Algeria from 1950 to 2004. The Russian Caucasus and some other regions in the World were considered for comparison [Smirnov 1993]. The autosimilarity parameters were calculated in aftershock zones for the space of the earthquake preparation, and included the determined number of seismic events of $M \geq 2$ during the time interval $\Delta T=1.5$ years. Each zone (earthquake preparation area) was divided into elementary volumes of linear dimension $\Delta x=(5-7) L_{i}$ and depth $\Delta z=20 \mathrm{~km}$. The area of the aftershocks constituted the boundary of the scaling field.

The recurrence plot slope $\gamma$ is calculated by the maximum-likelihood method [Zavyalov 2006]. A regional earthquake catalog of the Zemmouri zone that contained 1,414 events was studied following a check for homogeneity.

For the measure of the fractal dimension $D$ of a set of acoustic or seismic events, we used the cellular evaluation and correlation, and we interpreted the correlation dimension as the fractal dimension, assuming that the seismicity was near a homogeneous fractal.

The correlation dimension $D$ was calculated by applying the formula [Smirnov et al. 1995, Baddari and Frolov 1997]:

$$
D=\lim _{l \rightarrow 0} \frac{\log C(l)}{\log l}
$$

where $C(l)$ is the correlation integral, which is defined as:

$$
C(l)=N l \bar{r}-\bar{r}_{i}-\bar{r}_{j} l \leq \frac{l}{m(m-1)}
$$

where $N$ is the number of pairs of seismic events with distances between hypocenters that are less than $l$, and $m$ is the number of events. Evaluation of the correlation dimensions of the hypocenter set was carried out by plotting a histogram $C(l)$ and assessing the function of $\log C(l)$ and the regression estimate of the studied crustal area, limited below by the accuracy of the hypocenter evaluation, and above by the boundary of the assumed zone of strong earthquake generation, which was higher by an order of magnitude than the zone of the aftershocks.

\section{Results}

\subsection{Dynamics of the acoustic regime}

We consider the recurrence plot slope $\gamma$, the fractal dimension $D$, the relationship $D-3 \gamma$, and the crack concentration parameter $K_{s r}$ as parameters of the acoustic regime in these laboratory experiments. The variations in elastic-wave velocities and the electric parameters must, to a certain extent, mirror the stress-strain state of a rock and the development of anisotropy within it. 

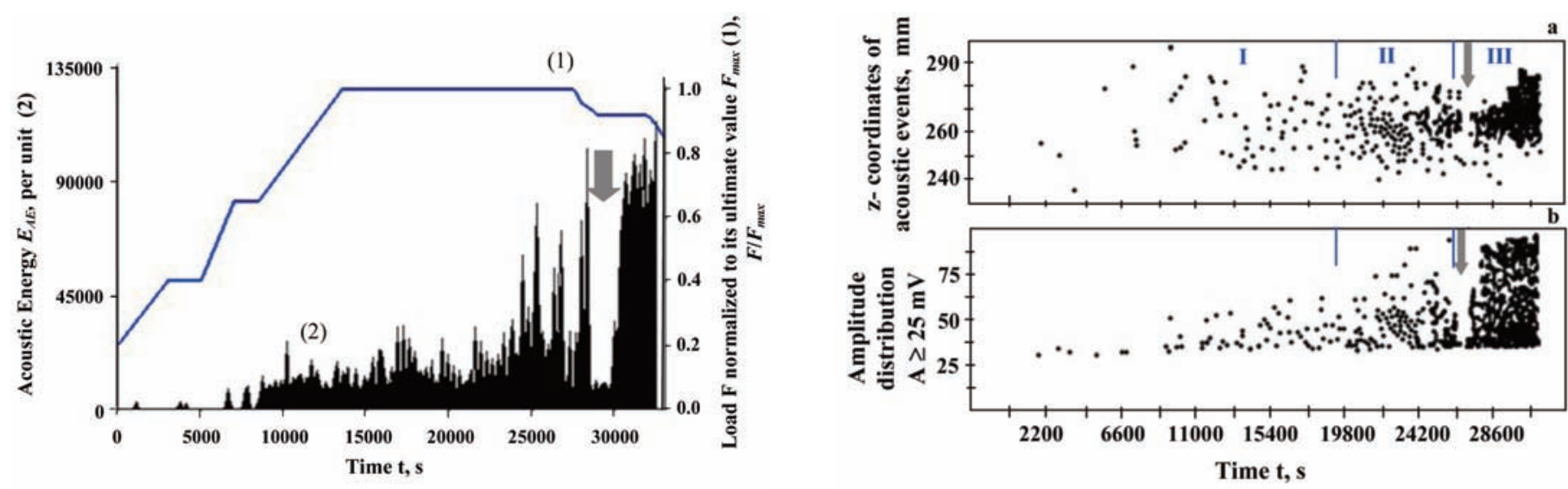

Figure 3 (left). Acoustic emission energy (1) and loading-cycle history (2) in experiment BF1. Arrow, acoustic quiescence.

Figure 4 (right). Distribution of $z$ coordinates (a) and signal amplitudes (b) of the acoustic events during the steps of the macrofailure process preparation in experiment BF2. Arrows, acoustic quiescence. I, II, III correspond to the stages of strike-slip fault generation.

Figure 3 illustrates the loading history and the acousticevent activity in experiment $\mathrm{BF} 1$, where the acoustic energy was summed in 50-s intervals. Figure 3 shows an acoustic quiescence at $t=28650-30400 \mathrm{~s}$, which corresponds to the scenario preceding the transition from the lower to the higher levels of cracking, and consequently to an avalanchelike crushing of barriers in the course of expanding failure. The distribution of amplitudes $(A \geq 25 \mathrm{mV})$ and $z$ coordinates of the acoustic event selected in experiment BF2 (Figure 4) shows the initial dispersive character, followed by a localization and an increase in the maximum amplitudes over time. Phase I recorded a maximum value up to $(t=19800 \mathrm{~s})$ of the loading process and developed a dispersive distribution, which was sparse with noncorrelated microcracks. The acoustic events generated are random in the rock block, and do not affect one another during this step. In phase II $(t=19800-27600 \mathrm{~s})$, we have a gradual concentration of the sources of the acoustic event in the small domain of the central part to the left of the block formation of a fractal structure of acoustic regime that leads to an increasing mutual effect of the acoustic events. Phase III $(t=27600-30200 \mathrm{~s})$ was characterized by evolution of the microcracks for the triggering of the main failure, which appeared on the surface of the block. The acoustic quiescence corresponded to the expansion of the fractal structure from one scale level to a greater one.

Figure 5 shows the variations of the recurrence plot slope $\gamma$, the fractal dimension $D$ and the relationship $D-3 \gamma$ during the block deformation in experiment $\mathrm{BF} 1$, and the normalized curve of the acoustic activity $N / N_{\max }$. Time $t$ is normalized to its final value (time of macrofailure creation $t_{f}$ in the laboratory experiment). Three stages of variations of the acoustic rate can be noted: I) rise in the acoustic intensity, marked by weak variations in $\gamma$; II) acoustic quiescence, marked by a decrease in $\gamma$; and III) renewed intensity prior to failure, which is indicated by an arrow, and a decrease in $\gamma$ to 0.4 . The parameter $D$ changes from a mean value of 2.89 in stage II, to 1.1 at the final stage III of loading. $D \geq 2.5$ corresponds to a quasi uniform separation stage of the microrupture in the rock. The decrease in $D$ to 1.1-1.5 shows the formation of a fractal structure of defects at the time of stress drop, when the rock loses its reserves in elastic energy. During stage I $(D-3 \gamma>0)$ there is a gradual accumulation of microcracks. Stage II $(D-3 \gamma \approx 0)$ is noted in the area of maximal stress, and takes up most of the microcracks and the beginning of their interactions after reaching a critical concentration in the area of the future macrofracture. Stage III $(D-3 \gamma<0)$ is marked by progression of the main macrofailure. The acoustic-event energy in phase III is four-fold that in phase I. The number of cracks in phase III, which is characterized by high acoustic activity and stress diminution, must be three-fold less than that in phase I, according to the recursive law of acoustic events. According to the generalized dependence between the rupture size $L_{i}$ and the energy $E_{s}$ of an earthquake [Riznichenko 1976, Sobolev and

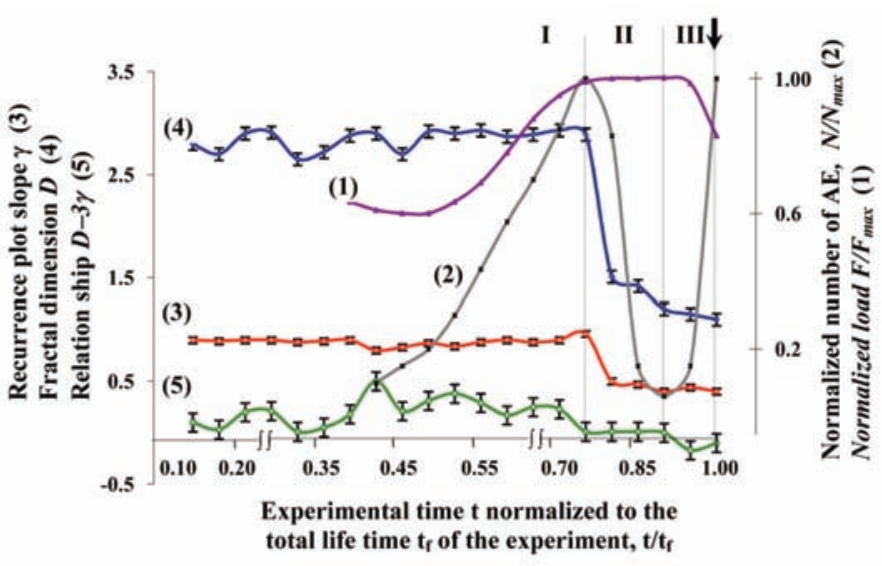

Figure 5. Dependence of the load $F / F_{\max }(1)$, the acoustic emission $N / N_{\max }$ (2), the recurrence plot slope $\gamma(3)$, the fractal dimension $D(4)$, and the relationship $D-3 \gamma(5)$ on the normalized time during the bloc deformation in experiment BF1. Arrow, time when the factures reached the free surface. I, II, III, correspond to the stages of strike-slip fault generation. 

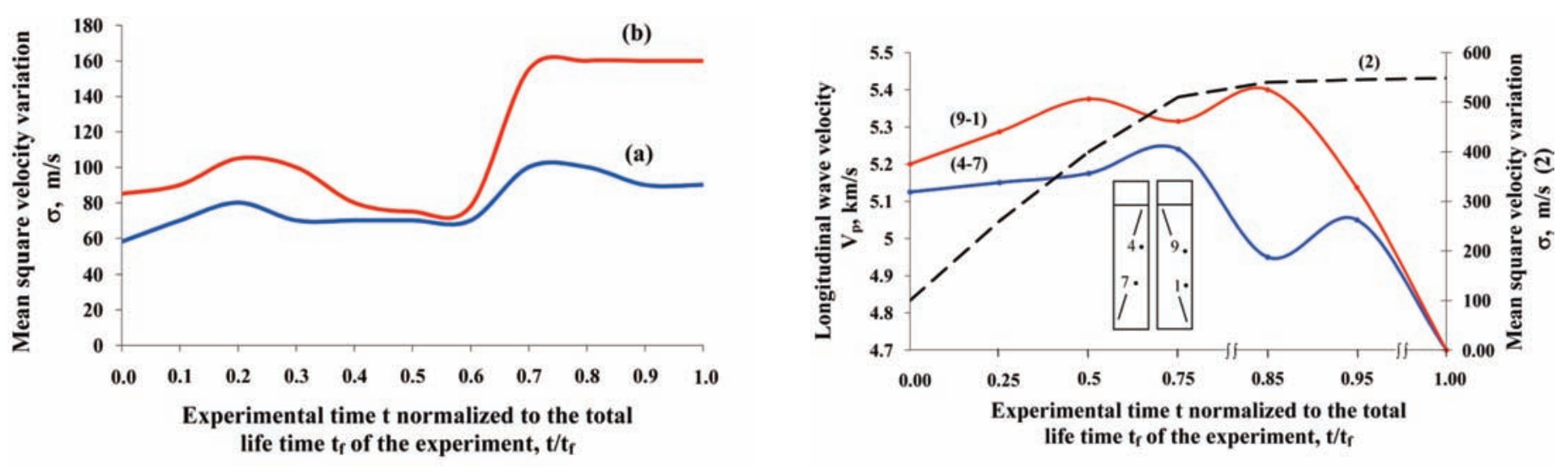

Figure 6 (left). Dispersion in integral wave velocity in the sample measured in the direction $x$ (a) and $y(b)$ as functions of the normalized time through the block deformation in experiment BF2.

Figure 7 (right). Example of $P$-wave velocity for different sounding of crack routes (9-1) and (4-7), and the corresponding dispersion measured for 12 routes (2) as functions of normalized time in experiment BF2. The sounds 9, 4 were located on side I, and 7 and 1 on side III. Inset: the diagram of the positions of the stress concentrators and the ultrasonic sounds.

Ponomarev 2003]:

$$
\log L_{i}(\mathrm{~km})=0.244 \log E_{s}(j)-2.266
$$

the elastic pulses energy radiated by separate cracks during stage III is about four-fold higher than that in stage I. The decrease in $\gamma$ and the fractal dimension $D$ are explained by the formation of a fractal structure in an acoustic regime of major rank in the rock. As a rule, stages (I-II) in all of the experiments that were marked by high $\gamma$ and parameter $D$ values were followed by a period of reduced $\gamma$ and $D$, when the macrorupture occurs in the last stage. This decrease in the recurrence plot slope $\gamma$ and the fractal dimension $D$ are caused by coalescing cracks and the formation of larger cracks. An anomalous decrease in the slope of the recurrence plot and the fractal dimension can be regarded as one of the precursors of an approaching macrofailure.

\subsection{Variations in elastic-wave velocities}

The analysis of the velocity variations in the experiments (BF1, BF2, BF4) shows that if a zone is characterized by a low velocity, then a domain of high velocity will be formed around it, and vice versa. It was found that above about a half of the failure strength, the average velocity became a non-linear function of the load. In particular, the velocity reached a maximum when the loading was half the maximal, possibly due to the developing fractal structure. Figure 6 illustrates the values of the mean square velocity deviation $\sigma$, measured along the $x$ and $y$ axes at different loads in experiment BF2. The calculation was performed for the 12 paths in each direction. The velocity variance increased significantly for loads increasing from about $0.7 t / t_{f}$ up to the final macrofailure load.

Anisotropy was recorded in the velocities in the directions $x$ and $y$, parallel and perpendicular, respectively, to the direction of the main load F. A considerable degree of elastic anisotropy developed prior to failure. This is due to the oriented microcracks that have a major role in the failure process itself. The average velocities before loading were $V_{p(X)}$ $=5.36 \mathrm{~km} / \mathrm{s}$ and $V_{p(Y)}=4.98 \mathrm{~km} / \mathrm{s}$. After the loading, these became $4.72 \mathrm{~km} / \mathrm{s}$ and $4.51 \mathrm{~km} / \mathrm{s}$, respectively. A typical example of the anisotropy of the $P$-wave velocity is shown in Figure 7 . The velocity anisotropy observed was associated with changes in the microcrack density and distribution pattern. We noted an increase in these prior to the macrofailure. The maximum of this increase coincided with the dynamic propagation of the fractal structure. The mean square velocity $\sigma$ deviation determined for 12 routes shows that the increase in stress is accompanied by increased scatter in the velocities. Figure 8 demonstrates the behavior of the ratio $V_{P} / V_{S}$ velocity variations for sets of elements 2-3 and 6-8 between the stress concentrators. It can clearly be seen that in the part 6-8 of the block, $V_{P} / V_{S}$ increases at $t=0.85 t / t_{f}$ and decreases up to $0.95 t / t_{\rho}$ In the element 2-3, $V_{P} / V_{S}$ decreases at $0.5 t / t_{f}$ increases at $0.85 t / t_{f}$, and decreases up to $0.95 t / t_{f}$, i.e.

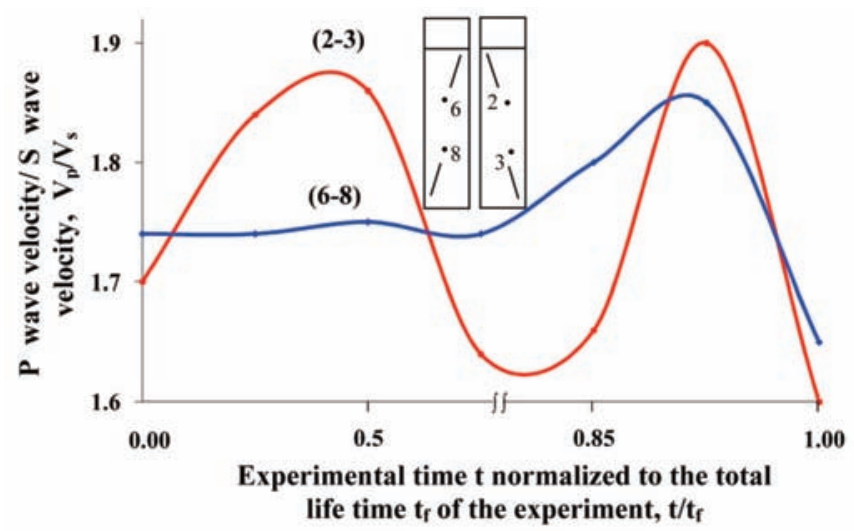

Figure 8. Typical anisotropy in the variation of the ratio $V_{p} / V_{s}$ for sets of routes between the stress concentrators in different sounding directions (as indicated) in experiment BF2. Inset: the diagram of the positions of the stress concentrators and the ultrasonic sounds. 
the dynamics of the fractal structure formation are more active than in direction 2-3 of the block. The middle part of the block is a zone of intense cracking, and in the upper left part of the block the cracking is practically negligible. To sum up, the velocity contrast in different regions of the block increases failure, and the rupture occurs differently in the various regions of the intersections of these paths. The $S$-wave velocity appears to be more influenced by the microfailure process than the $P$-wave velocity. In addition, the $S$-wave velocity increase prior to failure is larger, and the decrease after failure is less than that of the $P$-wave during the microcracking stages. Overall, for the ultrasound traces under the angles of $40^{\circ}$ to $45^{\circ}$ with respect to the vertical sample axis (Figure 8 , direction 2-3), $V_{P} / V_{S}$ increases from 1.73 to 1.85 during the first stage of reversible deformation, decreases from 1.85 to 1.62 during the step of dispersed opening microcracks, increases again from 1.62 to 1.90 when the microcracks affect one another, and finally decreases to 1.6 in the last stage of macrofailure expansion. The ratio $V_{P} / V_{S}$ did not show significant changes in the stable zones. The ratio $V_{P} / V_{S}$ allowed the study of the mechanical anisotropy in the strainstress state of the block over definite intervals. The contrast in the behavior of $V_{P} / V_{S}$ appears to be evidence of numerous minor oriented cracks arising during the earlier stage of failure generation. The decrease in $V_{P} / V_{S}$ in all cases during the final stage testifies to the process of big crack formation.

\subsection{Electric parameter variations}

Local variations of apparent resistivity $\Delta \rho_{a}$ were detected, and in certain zones, resistivity anisotropy developed over time. The anisotropy detected at the initial stage of loading is associated with the failure of the pre-existing defects. As the stresses increased, the portion of progressively smaller defects among the failed defects increased. In Figure 9, it can be seen that the greatest variations are recorded in the first step of the loading cycle, at (0.25-0.45) $t / t_{p}$, where their relative value reaches $40 \%$ of the variations of the apparent resistivity. Anisotropy in $\Delta \rho_{a}$ was recorded in the second, at (0.6-0.8) $t / t_{p}$, and the last, at (0.8-1) $t / t_{f}$, stages of rock failure, and can be qualitatively explained by the development of oriented sets of cracks. This is confirmed by the increase in the acoustic activity in that region between the stress concentrators during the final stage of macrofailure expansion. The decrease in $\Delta \rho_{a}$ in one direction of the rock block might be due to expansion of a conducting path system in this direction during crack accumulation. The increase in $\Delta \rho_{a}$ in the other perpendicular direction is probably caused by the coalescing oriented cracks and the rupture of the conducting path system during genesis of the macrocrack.

As the block was loaded, the spontaneous electric field $\Delta U$ changed significantly. We recorded some cases of initiation and disintegration of local electric anomalies when the load varied in the time range (0.5-0.9) $t / t_{f}$ Figure 10 shows

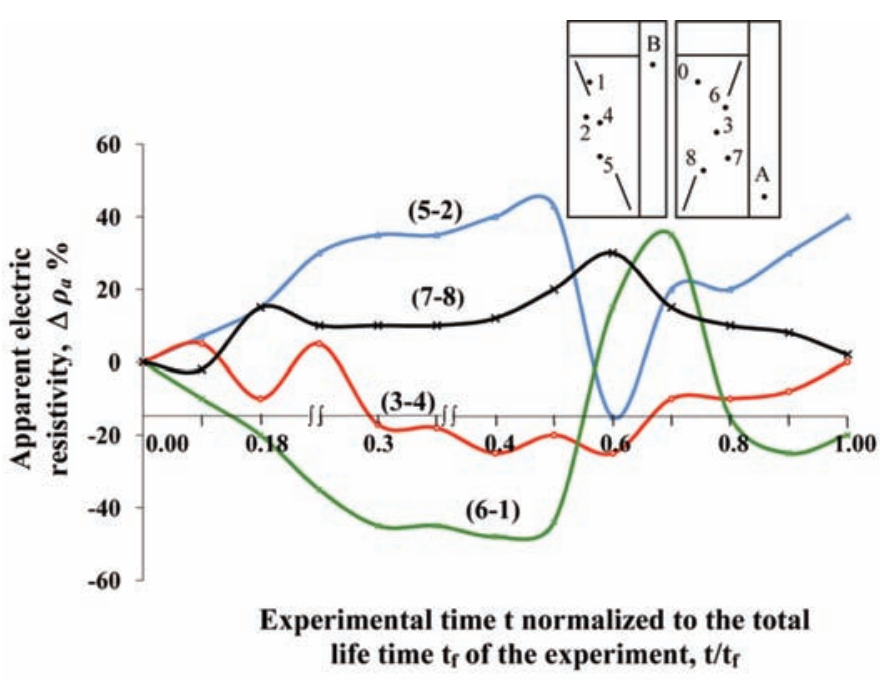

Figure 9. Anisotropy registered in apparent electric resistivity measures for different positions of pick-up electrodes pairs (as indicated) in experiment BF2. Inset: the diagram of the positions of the stress concentrators and the electrodes. 0, zero electrode; A, B- feeding electrodes.

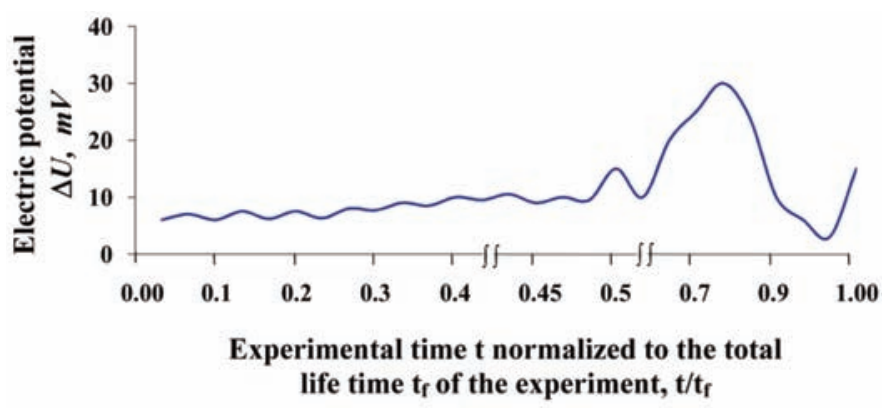

Figure 10. The local anomaly of the electric potential registered prior to macrofailure in experiment BF2, for the electrodes going through the barrier.

one of the anomalies that began at $0.5 t / t_{f}$, and reached a maximum of about $30 \mathrm{mV}$ at the beginning of final stage at $0.8 t / t_{f}$ Its life-time changed from 25 to 55 minutes, the reason probably being due to an electric relaxation phenomenon.

After loading, the variations of surface electric field significantly decreased and nonlinear relationship between mechanical and electric fields has been obtained. The disappearance of anomalous regions during load removal occurred with a lag of several tens of minutes. A number of features observed in the electric disturbances appear to be explainable qualitatively within the framework of ionic migration mechanisms. It is apparent that under high strain the dynamics of increase and of relaxation of the electric potential $\Delta U$ (in the last stage of the unstable deformation) does not correlate with the variation of the load, which in our opinion reflects the irregular process of failure in the rock.

\subsection{Crack concentration parameter}

In the laboratory experiments, the crack concentration parameter $K_{s r}$ diminishes significantly in phase III in the interval (0.7-1) $t / t_{f}$ before the macrorupture (Figure 11). For important values of $K_{s r}$, the cracks do not interact among 


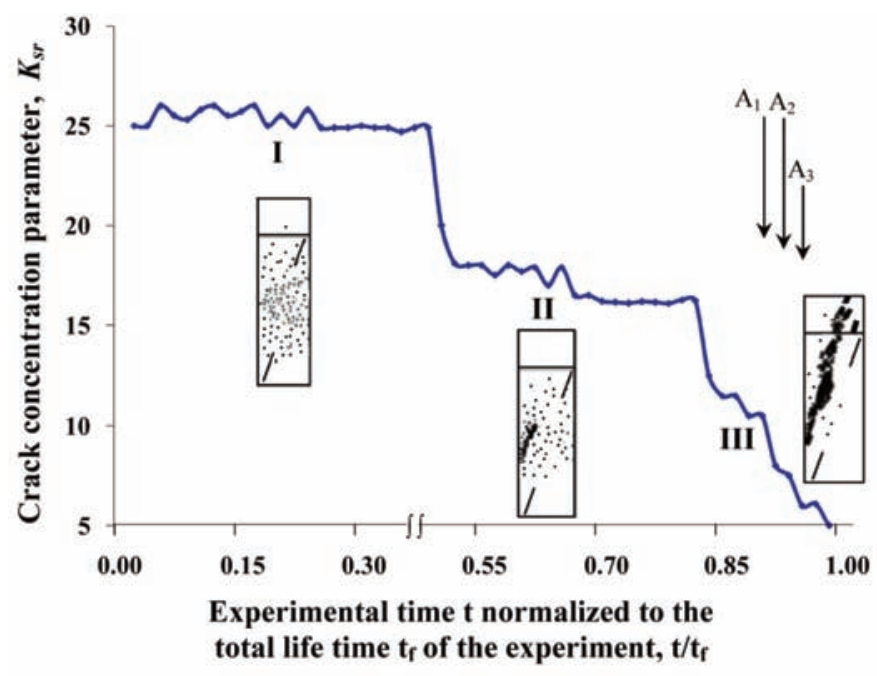

Figure 11. Variations in the parameter $K_{s r}$ during the block deformation in experiment BF1. The arrows denote amplitudes: $A_{1}, A=80 \mathrm{mV} ; A_{2}, A=83 \mathrm{mV}$; $A_{3}, A=85 \mathrm{mV}$. I, II, III, correspond to the stages of macrocrack formation.

themselves. Starting with the second stage in the range of 0.5-0.7 t $/ t_{f}$, a gradual clustering of the acoustic events within a small portion in the core can be detected. When the cracks are concentrated and their density becomes critical $\left(K_{s r} \approx 3-7\right)$, i.e. when the crack concentration reaches a stage where the mean relative distance between the cracks is $3-7$, a rupture is formed, which causes relatively quick failure of the body, and the deformation process moves to the progressive damage phase of the material. This leads to the formation of a fractal structure in the acoustic regime.

4.5. Temporal variations in the structure of a seismic regime

Analogous changes have been recorded for the recurrence plot slope $\gamma$ in studies in various areas of the Algerian seismoactive regions. The $\gamma$ decreased from 0.64 to 0.4218 months before the seismic event of Zemmouri $(M=$ 6.9) of 21 May, 2003, and from 0.68 to 0.3820 months before the seismic event of El Asnam ( $M=7.3)$ of 10 October, 1980 (Figure 12). The decreases are significant at a level of 0.05 .

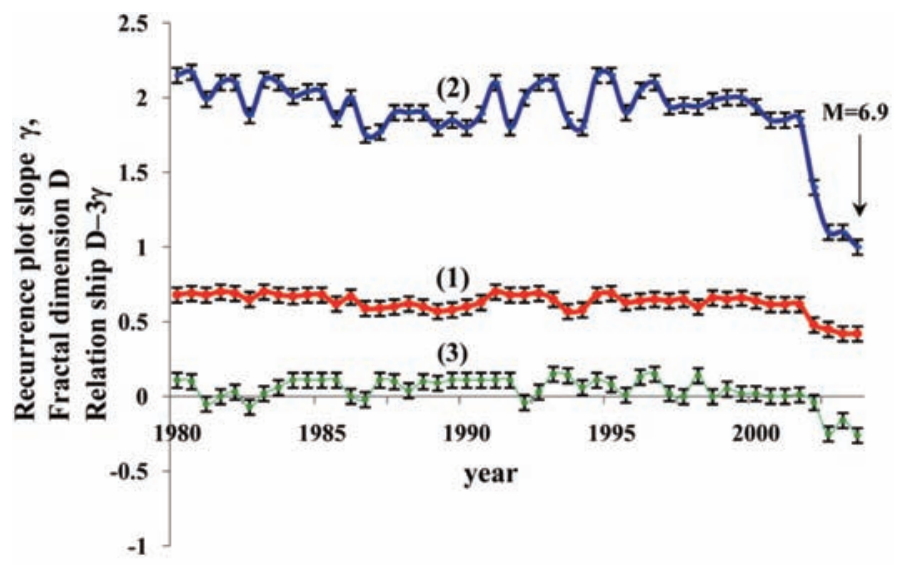

Figure 12. Variation of the seismo-acoustic self similarity parameters $B$-value (1), fractal dimension $D(2)$, and relationship $D-3 B$ (3) prior to the Zemmouri earthquake $M=6.9$.
The Izmit earthquake in Turkey $(M=7.4)$ of 17 August, 1999, had a decrease in $\gamma$ from 1.2 to 0.78 with respect to the normal conditions [Zavyalov 2006]. The seismic event of Vanskoy $(M=7.0)$ of 24 December, 1976, and of Spitak $(M=6.9)$, of 7 December, 1988 [Smirnov 1993], were characterized by decreases in $\gamma$ to 0.32 before the main shocks.

Therefore, we can say that $\gamma$ is among the main indices for the creation of a macrorupture at the corresponding scale. The $\gamma$ remains constant or increases at the beginning of the anomaly, and then decreases until the triggering of the macrofailure (block fracture, earthquake).

The earthquakes of El Asnam and Zemmouri showed a decrease in the fractal dimension $D$ from 1.5 to 1.2 and from 1.62 to 1.25 , respectively, 15 and 18 months before the main events (Figure 12). The average value for the $D$ obtained for two areas was none other than about $1.52 \pm 0.05$. Analogous variations in parameter $D$ preceded the earthquakes of Spitak and Vanskoy by 12-18 months, from 1.5 to 1.45 and 1.4 to 1.2 , respectively. The decrease in $D$ can be explained by the progressive appearance of local instability in the deformed volume of the materials as these approached macrocracks.

\subsection{Variations in the crack concentration parameter}

in the seismoactive regions

The crack concentration parameter $K_{s r}$ decreased to 4.35 and 7.6, respectively, 8-10 months before the main seismic events of El Asnam and Zemmouri (Figure 13). $K_{s r}$ reached 4.2 before the earthquake of Kamchatka of 5 December, $1997(M=7.9)$ [Zavyalov 2006]. In other words, when the parameter $K_{s r}$ reached the critical value $4-7$, the crack clustering acquired a threshold character that resulted in relatively quick fault expansion. The quasi-invariance of $K_{s r}$ for zones which are far from epicenters was also noted, which confirms the hypothesis of spatial localization of seismic events at the level of the rupture zone. The values of $K_{s r}$ before the macrorupture calculated for the geophysical medium at different scales are in the interval 3-7.

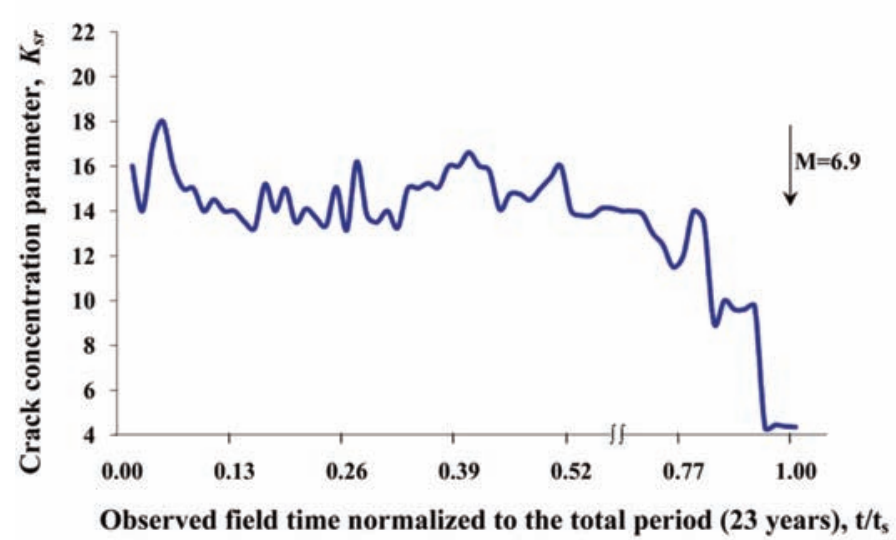

Figure 13. Dependence of the parameter $K_{s r}$ on the normalized time in $200 \times 200 \mathrm{~km}$ zone that contained the epicenter of Zemmouri earthquake $M=6.9 . t_{s}$ corresponds to 23 years of the experimental field time. 


\section{Discussion and comments}

\subsection{Interactions of acoustic events and formation of fractal structure}

The chosen experimental technique in the laboratory allowed the extension of the evolution phase of the rock macrorupture. The formation of fractal structures developed during the deformation of the rocks at different scales of change over time. The fractal structure created during the phase of the stress drop, when the rock loses its reserves of elastic energy, is conditioned by the evolution of its elements (the microcracks). It is the precursor of the genesis of the macrofailure. During the first step of rock deformation, microcracks appear to be homogeneously distributed, and as microfailures progress, interactions between them take place. This leads to their coalescence and initiation of the fractal structure, which is marked by the variation of the acoustic regime. The formation of a fractal structure leads to a critical diminution in the auto-similarity parameters $\left(\gamma, D, K_{s r}\right)$ and creates anisotropies in the elastic and electrical properties of the rock sample. Amitrano [2006] showed that the process of damage localization is related to the passage from isotropic uncorrelated damage to anisotropic correlated damage.

The anisotropy of the strength and the distributions of the mechanical stresses in a deformed rock massif are often a reason for local dominance of brittle destruction, which results in catastrophic phenomena (earthquakes, rock bursts, collapses, etc.). For any rock, there is always some strength distribution of the contacts between the grains, which are variable in the massif volume. The process of deformation is accompanied by rupture of some parts of the least strong contacts, and alteration of their distribution in microfracturing systems. Both zones of increased stiffness and zones of increased quasi-ductility are created, and the process of deformation of the anisotropy of the elasticity and the strength increases. This transformation is a result of the reorganization in the rock of ionic and electronic physical subsystems, which must lead to characteristic changes in the mechanical and electrical properties, depending on the stress-strain state of the rock. These studies have allowed us to show that this process of failure involves three stages: dispersed deformation, and initiation and expansion of the fractal structure.

The fractal dimension $D, \gamma$ and the crack concentration parameter $K_{s r}$ all decrease before the main seismic and acoustic event of the studied geophysical field, at different scales. These decreases are of $20 \%$ to $80 \%$ for $\gamma, D$ and $K_{s r}$ with respect to their usual values with 6 to 18 months of field observations before the main seismic shock $(M \geq 6)$ and 30-60 minutes, i.e. (0.9-0.95) $t / t_{\rho}$, before the macrofailure in the rock sample in the laboratory experiments. We did not record such an anomaly for seismic events of $M<5$ in this Algerian seismoactive region. The decrease in $D$ preceded the decrease in $\gamma$ at different scales of the medium. The parameters $D, \gamma$ and $K_{s r}$ of the geometric

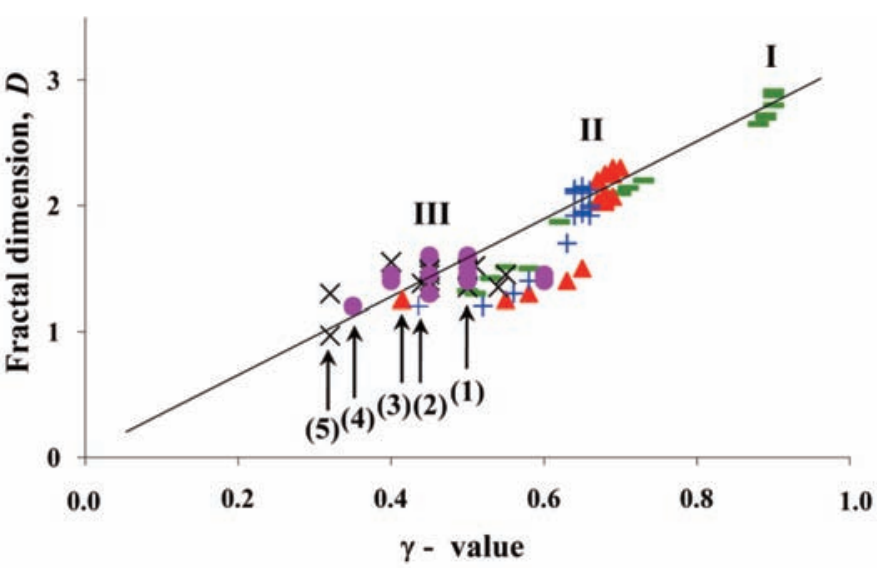

Figure 14. Dispersion diagram of parameters $\gamma$ and $D$ in experiment BF3; $\Delta$ Zemmouri; + El Asnam; $\times$ Vanskoy; $\bullet$ Spitak; / $D=3 \gamma$, arrows indicate (1) the macrofailure in the block sample; (2) El Asnam earthquake ( $M=7.3)$; (3) Zemmouri earthquake $(M=6.9)$; (4) Spitak earthquake $(M=6.9)$; (5) Vanskoy earthquake $(M=7.0)$. I, II, III, correspond to the stages of seismo-acoustic regime evolution.

set of acoustic events or seismic events reflect the variations in the seismo-acoustic regime. The decreases in $\gamma, D$ and $K_{s r}$ are associated with the gradual clustering and the expansion of the fractal structure in the geophysical medium under load. The existence of a critical crack density in the transition from dispersed volumetric to localized failure testifies the rise in the unstable deformation in the failing parts of the medium.

\subsection{Temporal variation}

in fractal structures in a seismo-acoustic regime

The distribution $(\gamma, D)$ of the seismo-acoustic data can be adjusted by the line $D=3 \gamma$ (Figure 14). The evolution of the acoustic regime is directed from cluster I to clusters II and III. Cluster I corresponds to microcrack nucleation when the stresses are increased in the rock. Cluster II corresponds to the phase of the accelerated accumulation of microcracks in different parts along the future rupture band of the rock, and to their critical concentration and stress drop. This transition reflects the appearance of a fractal structure in the acoustic regime, with a simultaneously corresponding decrease in the parameters $\gamma$ and the fractal structure $D$. Cluster III corresponds to the progressive evolution of damage and the formation of the mean macrofailure when the stress continues to drop. Cluster I is absent at the scale of seismoactive regions characterized by low values of fractal dimension $D$ and of $\gamma$. This is probably due to the formation in the Earth crust of a fractal structure that is more developed than that obtained in the laboratory. The relationship $D=3 \gamma$ means that $\zeta=0$ (Equation 14). The interruption of this equivalence $(\zeta \neq 0)$ can be related to the redistribution of the medium stress-strain state as a function of the dimension of the rupture and of the seismo-acoustic regime evolution preceding and succeeding the mean seismic event (acoustic even) [Deng et al. 2009, Liu et al. 2009]. Deviation over time from the relationship $D-3 \gamma=0$ can be seen as a variation in the stable state of the seismo-acoustic regime. 


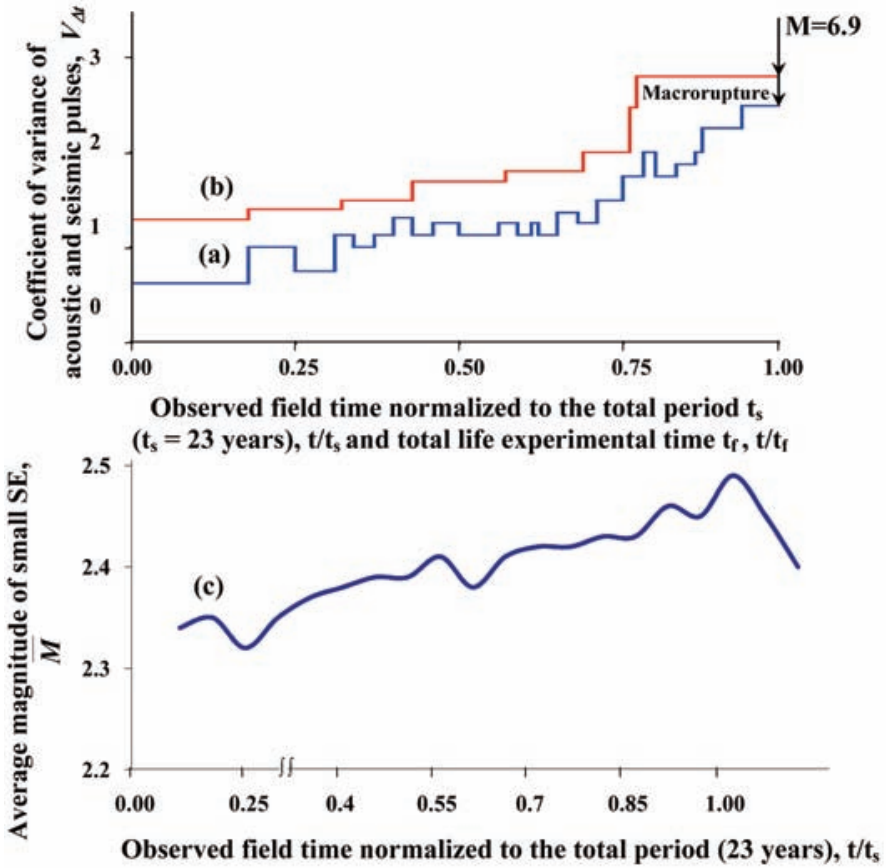

Figure 15. Top: Changes in the variance coefficient of the number of acoustic and seismic pulses during the expectation period of an important energetic event respectively for the rock block (a) (experiment BF4) and the Zemmouri earthquake (b). Bottom: Average magnitude $\bar{M}$ of small earthquakes in the Zemmouri area (c). $t_{s}$ and $t_{f}$ correspond to the experimental field time ( 23 years) and the total life time of the laboratory experiment, respectively.

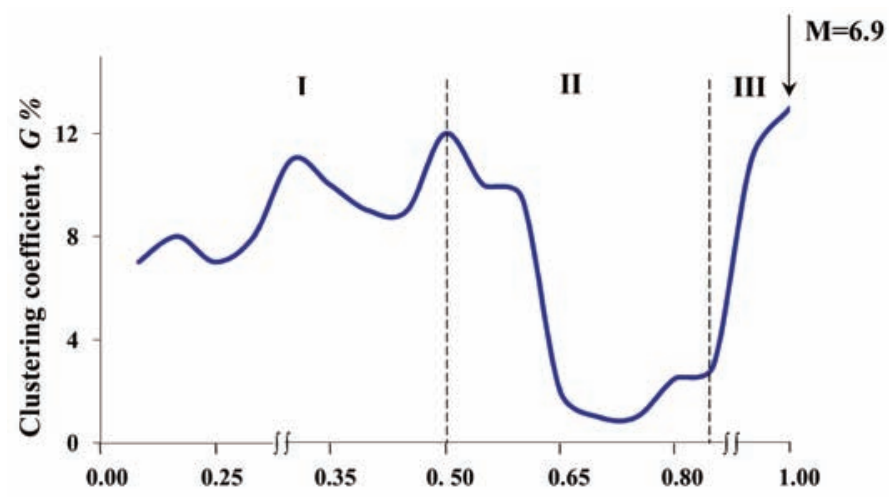

Observed field time normalized to the total period ( 23 years), $t / t_{s}$

Figure 16. Changes in the percentage of the clustering earthquakes $G \%$ in the 2003 epicentral area of the Zemmouri earthquake, and prior to the Zemmouri earthquake $M=6.9$. I, II, III, correspond to the earthquake stages preparation.

\subsection{Changes in the percentages}

of the clustering seismo-acoustic events

Figure $15 \mathrm{a}, \mathrm{b}$ shows the variation of the variance coefficients of the number of acoustic events and seismic events during the waiting period of major strong events at different scales. We note the analogy in the increasing of $V_{\Delta t}$ until the triggering of this event. Therefore, the gradual reinforcement of ruptures in the epicentral zone is accompanied by an increase in the average magnitude $\bar{M}$ of the small seismic events that preceded the mean shock in the Zemmouri earthquake area (Figure 15c).
The acoustic and seismic events have spatial hierarchy similitude. The three stages of the deformation of the geophysical medium mentioned above are manifest in the variation of the clustering coefficient $G \%(G \%$ is the ratio of the number of clustering small earthquakes of $M_{\min } \geq 2$ in the epicentral area of the Zemmouri earthquake, with their total number $\sum N$ in the studied seismogenic zone; $G \%=$ $\mathrm{Ng} / \sum \mathrm{N}$ ) [Baddari 1997, Sobolev and Ponomarev 2003] (Figure 16). We have considered that two successive seismic events are clustered if the critical distance $d_{c r}$ and the critical time $t_{c r}$ between them is less than the critical values corresponding to these two parameters. For Algeria, we have used the following empirical correlations [Sobolev 1995, Baddari et al. 1996): $t_{c r}$ (hours) $=a 10^{b \mathrm{M}}$ and $d_{c r}(\mathrm{~km})=c L_{i}$, where $a=5.2 \times 10^{4} ; b=0.75 ; c=3$, as the cluster model parameters determined as coefficients of linear regression for the functions $t_{c r}$ and $d_{c r} ; L_{i}$ is calculated from Equation (15), and it is the length of a rupture in the epicenter of an earthquake of a given energy; $M$ is the magnitude of the first earthquake in a pair of seismic events. The increase in $G \%$ in stage I (Figure 16) is due to an increase in the interaction between fractures in the epicentral area. The decrease in $G \%$ during stage II is caused by the strain and stress relaxation. The increase of G\% during stage III is explained by the coalescing of all of the fractures and the formation of the seismic source of the 2003 Zemmouri earthquake.

\subsection{Hierarchical arrangements}

of adjacent rupture sizes at different scales

After the experiments, the microscopic study of the residual ruptures showed adjacent microcracks of ranks, respectively: $32 ; 11 ; 3.5 ; 1.15 ; 0.33 \mathrm{~mm}$ in experiment $\mathrm{BF} 1$, and $42 ; 12 ; 5 ; 2.5 ; 0.8 ; 0.35 \mathrm{~mm}$ in experiment $\mathrm{BF}$. The ratio of the two basic adjacent sizes in both of these experiments is $L_{i} / L_{i-1} \approx 2-3.5$. As a result, the $L_{i} / L_{i-1}$ ratio was close to $2-4$ in all of the experiments. For the region of Zemmouri, a rupture hierarchy was brought out by analogy, i.e. $L_{1} \approx 80 \mathrm{~km} ; L_{2} \approx 40$ $\mathrm{km} ; L_{3} \approx 10 \mathrm{~km} ; L_{4} \approx 3 \mathrm{~km}$. The presence of active faults in the Zemmouri seismoactive region gives rise to an intricate hierarchical structure of the stress field. An ordered hierarchical pattern of active spatial fault distribution in central Asia was realized by Ulomov [1991]. The ratio of the average geometrical sizes of adjacent fault ranks was close to 3. In agreement with Sadovsky et al. [1991] and Sobolev, [1995], the average ratio of the adjacent basic dimensions of the seismogenic blocks $L_{i}=/ L_{i-1} \approx 3.33$ constitutes a highprobability real fact of nature, with the value in keeping with the scale, from fractions of a micron to hundreds of kilometers. The deformation of the geophysical field is related to the continuation in the accumulation of energy, which differs from the active zone to another seismic zone according to the motion speed of the block rocks, and the dissipation speed of the energy at different hierarchy levels of the medium. 


\section{Conclusion}

The seismo-acoustic process is a discrete stochastic process that is related to the kinetic accumulation of ruptures. This suggests that the development of the failure process is controlled by the same physical mechanism. The process of rock failure at different scales observed in the laboratory and the field experiments gives rise to a fractal structure that is due to the active interactions of cracks. The genesis and evolution of a newly formed fractal structure leads to anisotropy in the elastic and electric properties of the deformed geophysical medium, and to the appearance of anomalies in auto-similarity seismo-acoustic parameters (the slope of the recurrence plot $\gamma$, the fractal dimension $D$, the relationship $D-3 \gamma$, and the crack concentration parameter $K_{s r}$ ) during the last phase before the triggering of the mean seismo-acoustic event. The $\gamma$ and the fractal dimension $D$ decrease, their relationship $D \approx 3 \gamma$ is interrupted, and the parameter $K_{s r}$ of the crack concentration decreases to 3-7 during the expansion of the fractal structure. The decrease in the fractal dimensions at the time of the strong Algerian seismic events can be interpreted as a decrease in the dimensions of the seismic area, which is in agreement with the concept of failure localization during the period of an earthquake rise. Anomalous changes in the parameters $\gamma, D$, $D-3 \gamma$ and $K_{s r}$, on whatever scale, can be interpreted as one of the precursors of an approaching large dynamic event.

The acoustic rate consists of three main steps: dispersive releasing of the acoustic events; critical concentration of the acoustic events; and formation of the fractal structure; finally, there is the evolution of this fractal structure to give birth to the main fracture.

The changes in the number of clustering $M \geq 2$ earthquakes in the 2003 Zemmouri epicentral area showed three corresponding factors to those noted in the laboratory experiments. The rise in the clustering parameter $G \%$ during the first stage is due to growing interactions of seismogenic faults that can act as local mechanical-stress concentrators. The second stage is marked by a decrease in $G \%$ due to the increasing mechanical instability caused by the influence of the macrofracture nucleation and the attenuation of the interactions of the neighboring stress fields in the epicentral area. The increase in $G \%$ in the third stage is caused by coalescing failures and the formation of larger ruptures that generated the 2003 Zemmouri main seismic source.

The self-similarity of the rock structures and certain aspects of the seismicity show considerable heterogeneity of the cracks, and the intercrack contact properties. The experiments reveal that the deformed rock is divided into separate blocks, according to echelons of cracks of different sizes and orientations. The presence of active cracks gives rise to an intricate hierarchical structure of the stress-strain geophysical field that can be characterized by its fractal properties.

The similarity recorded at different scales in the evolution of the fractal structure can be used to monitor the development of seismic fractures and to predict the superficial earthquakes of the Algerian seismoactive regions at intermediate terms.

\section{References}

Amitrano, D. (2006). Rupture by damage accumulation in rocks, Int. J. Fracture, 139, 369-381.

Baddari, K. and G.A. Sobolev and A.D. Frolov (1996). Similarity in seismic precursors at different scales, Comptes Rendus de l'Académie des Sciences, Série Geoscience, 323, 755-763.

Baddari, K. (1997). Experimental study of the structure fractal formation in the seismic process, Bulletin du Service Géologique de l'Algérie, 8, 171-182.

Baddari, K. and A.D. Frolov (1997). Modeling of fractal structure of geophysical field, Comptes Rendus de l'Académie des Sciences, Série Geoscience, 325, 925-930.

Baddari, K., G.A. Sobolev, A.D. Frolov and A.V. Ponomarev (1999). An integrated study of physical precursors of failure in relation to earthquake prediction, using large scale rock blocks. Annals of Geophysics, 42 (5), 771-787.

Baddari, K. and M. Djeddi (2009). Physics of the Earth, OPU, Alger, $388 \mathrm{pp}$.

Bizzarri, A. and M. Cocco (2006). A thermal pressurization model for spontaneous dynamic rupture propagation on a three- dimensional fault: 2 . Traction evolution and dynamic parameters, J. Geophys. Res., 111, B05304; doi: 10.1029/2005JB003864.

Deng, X.B., W.F. Du and G. Xu (2009). Physical model for rock burst based on acoustic emission. Controlling Seismic Hazard and Sustainable Development of Deep in Mines (RASIM7), 2 voll., 1597-1604.

Dresen G., S. Stanchits and E. Rybacki (2010). Borehole breakout evolution through acoustic emission location analysis, Int. J. Rock Mech. Min., 47 (3), 426-435.

Eftaxias, K., V. Sgrigna and T. Chelidze, eds. (2007). Mechanical and electromagnetic phenomena accompanying preseismic deformation: from laboratory to geophysical scale, Tectonophysics, 431 (1-4), 302 pp.

Gutenberg, B. (1956). The energy of earthquakes, Quarterly Journal of the Geological Society, 112, 1-14.

Han, F.S. and J.Y. Yang (2009). Numerical simulation for acoustic emission and source location of rock under triaxial compression. Controlling Seismic Hazard and Sustainable Development of Deep in Mines (RASIM7), 2 voll., 911-914.

Kanamori, H. (2004). The density of the physics of earthquakes, Proceedings of the Japan Academy, Ser B, 80 (7), 297-316.

Kasahara, K. (1985). Earthquake mechanism, MIR, Moscow, 264 pp.

Kuksenko, V.S., Kh.F. Makhmudov, V.A. Mansurov, U. Sultanov and M.Z. Rustamova (2009). Changes in structure of natural heterogeneous materials under deformation, J. Min. 
Sci., 45 (4), 355-358.

Lei, X., K. Masuda, O. Nishizawa, L. Jouniaux, L. Liu, W. Ma, T. Satoh and K. Kusunove (2004). Detailed analysis of acoustic emission activity during catastrophic farcture of faults in rock, J. Struct. Geol., 26, 247-258.

Lei, X. and T. Satoh (2007). Indicators of critical point behavior prior to rock failure inferred from pre-failure damage, Tectonophysics, 431 (1-4), 97-111.

Liakopoulou-Morris, F., I.G. Main, B.R. Crawford, B.G.D. Smart (2007). Microseismic properties of a homogeneous sandstone during fault nucleation and frictional sliding, Geophys. J. Int., 119 (1), 219-230.

Liu, J.P, Y.H. Li and Y.J. Yang (2009). Study of spatial distribution and fractal dimension of AE events during rock fracture. Controlling Seismic Hazard and Sustainable Development of Deep in Mines (RASIM7), 2 voll., pp165-170.

Lockner, D.A., J.D. Byerlee, V. Kuksenko, A. Ponomarev and A. Sidorin (1992). Chapter 1 Observations of Quasistatic Fault Growth from Acoustic Emissions, In: Fault Mechanics and Transport Properties of Rocks - A Festschrift in Honor of W.F. Brace, International Geophysics, 51, 3-31.

Lockner, D.A. (1993). The role of acoustic emission in the study of rock fracture, Int. J. Rock Mech. Min. Sci. Geomech. Abstr., 30 (7), 883-899.

Main, I.G., P.G. Meridith, P.R. Sammonds and C. Jones (1990). Influence of fractal flaw distribution on rock deformation in the brittle field, Geological Society, London, Special Publications, 54, 81-96.

Riznichenko, Yu.V. (1976). Crustal earthquake source size and the seismic moment, In: Earthquake Physics Studies, edited by Yu.V. Riznichenko, Nauka, Moscow, 9-27.

Sadovsky, M.A. and V.F. Pisarenko (1991). Seismic process and the block medium, Nauka, Moscow, 96 pp.

Sadovsky, M.A., L.G. Bolkhovitinov and V.F. Pisarenko (1991). Deformation of the Geophysical Medium and Seismic Process, Nauka, Moscow, 100 pp.

Senfaute, G., A. Duperet and J.A. Lawrence (2009). Microseismic precursory cracks prior to rock-fall on coastal chalk cliffs: a case study at Mesnil-Val, Normandie, NW France, Nat. Hazards Earth. Sys., 9 (5), 1625-1641.

Smirnov, V.B. (1993). Fractal features of seismicity of the Caucasus, In: Modeling of the Seismic Process and Earthquake Precursors, Russian Academy of Sciences, Moscow, 1, 121-130.

Smirnov, V.B. (1995). Earthquake repetition and the parameters of the seismic process, Vulcalnol. Seismol., 3, 59-70.

Smirnov, V.B., A.V. Ponomarev and A.D. Zavyalov (1995). Particularities in the formation and evolution of the acoustic regime structure in rock samples, Dokl. Acad. Nauk, $343,818-823$.

Smirnov, V.B. (1997). Spatial and temporal variations in parameters of seismic self-similarity, Vulkanologiya i seismologiya, 6, 31-41.
Smirnov, V.B. and A.V. Ponomarev (2004). Regularities in relaxation of the seismic regime according to natural and laboratory data, Physics of the Earth, 10, 26-36.

Smirnov, V.B., A.V. Ponomarev, P. Bernard and A.V. Patonin (2010). Regularities in transient modes in the seismic process according to the laboratory and natural modeling, Izv-Phys. Solid Earth, 46 (2), 104-135.

Sobolev, G.A. (1995). Fundamental of earthquake prediction, ERC, Moscow, $161 \mathrm{pp}$.

Sobolev, G.A., A.V. Ponomarev, A.V. Koltsov and V.B. Smirnov (1996). Simulation of triggered earthquakes in the laboratory, Pure Appl. Geophys., 147 (2), 347-355.

Sobolev, G.A. and A.V. Ponomarev (1999). Acoustic emission and preparation stages of failure in the laboratory experiment, Vulkanologiya i seismologiya, 4-5, 50-62.

Sobolev, G.A. and A.V. Ponomarev (2003). Earthquake physics and precursors, Nauka, Moscow, $270 \mathrm{pp}$.

Thompson, B.D., R.P. Young and D.A. Lockner (2006). Fracture in westerly granite under AE feedback and constant strain rate loading: nucleation, quasi-static propagation, and the transition to unstable fracture propagation. Pure Appl. Geophys. 163, 995-1019.

Ulomov, V.I. (1991). Zoning of Seismic Hazard, MaskanArkhit. Stroit. Uzbek. Kazakh. Azerb. Kyrgyz. Tadzhik. Turkmen., 9, 5-8.

Vettegren, V.I., V.S. Kuksenko, N.G. Tomilin and M.A. Kryuchkov (2004). Statistics of microcracks in heterogeneous materials (granites), Fiz. Tverd. Tela, 46 (10), 1293-1796.

Xu, S.C., X.T. Feng and B.R. Chen (2009). Acoustic emission characteristics and mechanical behavior of skarn under unaxial cyclic loading and unloading. Controlling Seismic Hazard and Sustainable Development of Deep in Mines (RASIM7), 2 voll., 435-440.

Youn, H. and F. Tonon (2010). Multi-stage triaxial test on brittle rock, Int. J. Rock Mech. Min., 678-684.

Yuan, R.F. and H.M. Li (2009). Identification of the predictive information before rock mass failure based on b-Value of ae events and its deficiency. Controlling Seismic Hazard and Sustainable Development of Deep in Mines (RASIM7), 2 voll., 1447-1450.

Zang, A. F.C. Wagner, S. Stanchits, C. Janssen and G. Dresen (2000). Fracture process zone in granite, J. Geophys. Res., $105,23651-23661$.

Zavyalov, A.D. (2006). Intermediate term earthquake prediction, Nauka, Moscow, 254 pp.

\footnotetext{
${ }^{\star}$ Corresponding author: Kamel Baddari, Laboratory of Physics of the Earth, UMBB, Algeria; email: doyenfs@umbb.dz.
}

(C) 2010 by the Istituto Nazionale di Geofisica e Vulcanologia. All rights reserved. 